\title{
Original
}

\section{Follicular thyroid carcinoma with skull metastases}

\author{
Maria Grazia Chiofalo ${ }^{1)}$, Sergio Venanzio Setola ${ }^{2)}$, Francesca Di Gennaro ${ }^{3)}$, Franco Fulciniti ${ }^{4}$, \\ Giuseppe Catapano $^{5)}$, Nunzia Simona Losito ${ }^{4)}$, Fabio Sandomenico ${ }^{2)}$, Orlando Catalano ${ }^{2)}$ and \\ Luciano Pezzullo ${ }^{1)}$ \\ 1) Thyroid and Parathyroid Surgery Unit, Istituto Nazionale Tumori, IRCCS Fondazione G. Pascale, via Mariano Semmola, 80131 \\ Naples, Italy \\ 2) Department of Radiology, Istituto Nazionale Tumori, IRCCS Fondazione G. Pascale, via Mariano Semmola, 80131 Naples, Italy \\ 3) Department of Nuclear Medicine, Istituto Nazionale Tumori, IRCCS Fondazione G. Pascale, via Mariano Semmola, 80131 \\ Naples, Italy \\ 4) Department of Pathology, Istituto Nazionale Tumori, IRCCS Fondazione G. Pascale, via Mariano Semmola, 80131 Naples, Italy \\ 5) Department of Neurosurgery, AORN G. Rummo, via Dell'Angelo I, 82100, Benevento, Italy
}

\begin{abstract}
Thyroid carcinoma with distant metastases at initial presentation, is uncommon. Skull metastases occur very rarely, with a reported incidence of 2.5-5.8\%. Here we report two cases of follicular thyroid cancer with skull involvement, and describe the diagnostic and therapeutic approach to metastatic thyroid cancer. We present the cases of a 70-year-old female and a 74-year-old female who presented with painless, large slow-growing masses of the skull. The patients underwent surgical excision of the skull masses, which were histologically diagnosed as metastatic follicular thyroid cancer, and total thyroidectomy, which confirmed the diagnosis of follicular thyroid carcinoma. They were treated with radioiodine and suppressive levothyroxine, which achieved local control of the disease. Management of metastatic thyroid cancer, requires a multidisciplinary approach and multimodality treatment. Distant metastases should be surgically removed whenever possible. Initial aggressive treatment is crucial in the management of metastatic thyroid carcinoma, providing the best chance to prolong patient survival.
\end{abstract}

Key words: Follicular thyroid cancer, Skull metastases, Bone metastases

FOLLICULAR and papillary thyroid cancers, generally referred together as differentiated thyroid cancer, are usually curable when detected at an early stage. Compared with papillary thyroid cancer, follicular thyroid cancer usually occurs in older patients and is more commonly associated with distant metastases and higher mortality [1]. The incidence of distant metastases at presentation ranges from $1 \%$ to $9 \%$ whereas distant metastases after initial treatment occur in about 7\% to $23 \%$ of patients [2]. The most frequent site of distant metastases is lung (49\%) and bone (24\%); multiple sites are involved in about $20 \%$ of cases. Although bone represents the second most common site of distant metastases, skull metastases are very rare.

Submitted Dec. 1, 2014; Accepted Feb. 4, 2015 as EJ14-0553 Released online in J-STAGE as advance publication Mar. 20, 2015 Correspondence to: Maria Grazia Chiofalo, M.D., Thyroid and Parathyroid Surgery Unit, Istituto Nazionale Tumori, IRCCS Fondazione G. Pascale, Via Mariano Semmola, 80131, Naples, Italy. E-mail: mgchiofalo@libero.it
The occurrence of distant metastases allocates patients older than 45 years, at stage IVC according to the American Joint Committee on Cancer criteria [3] and negatively affects survival either at initial presentation or after primary treatment, especially in patients older than 45 years for whom the five-year disease-specific survival approaches $35 \%[4,5]$. The management of metastatic thyroid cancer can be a hard challenge for clinicians, requiring a multidisciplinary approach and multimodality treatment.

Herein, we describe two cases of skull metastasis from follicular thyroid carcinoma at presentation and discuss the diagnostic and therapeutic modality.

\section{Methods}

\section{Case 1}

On April 2011, a 70-year-old female was admitted, to the neurosurgery department of another Institution for a painless, large slow-growing mass in the 
A

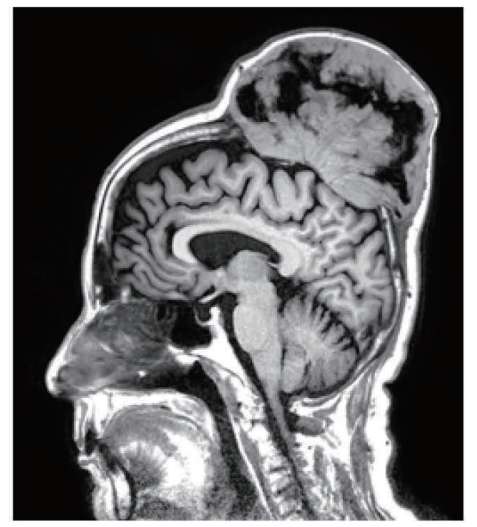

C

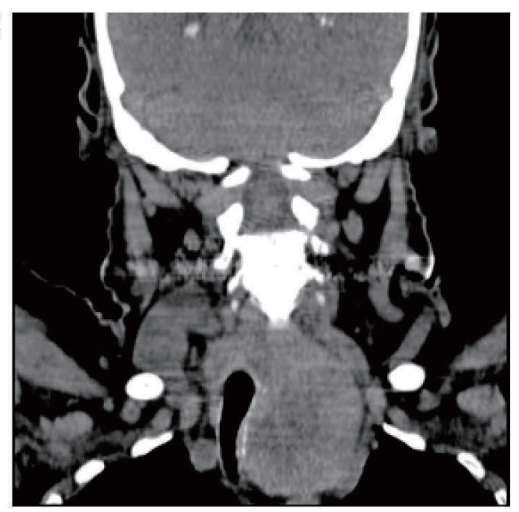

B

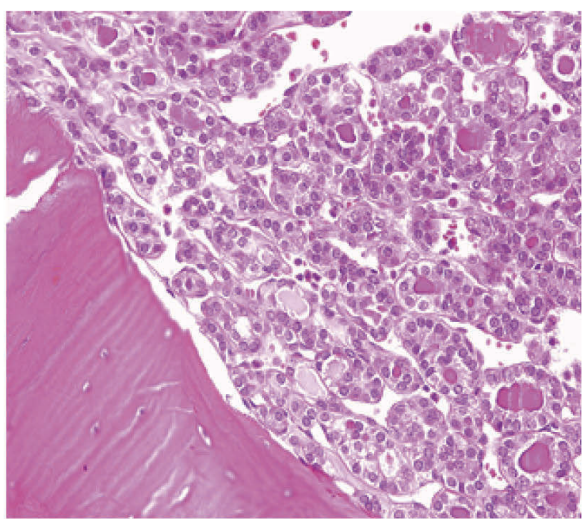

D

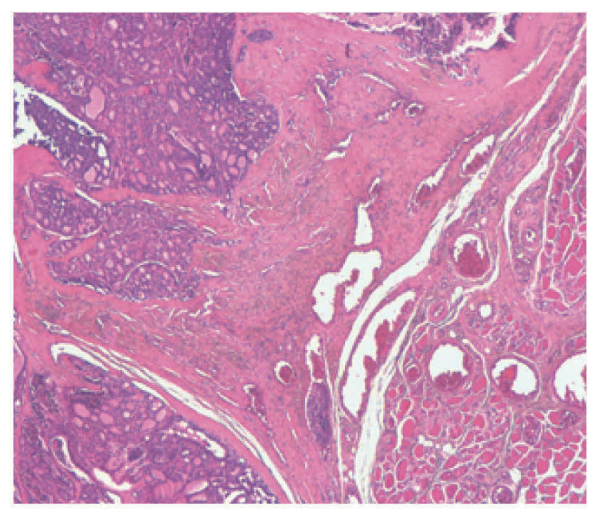

Fig. 1 Case 1

A. T1 weighted Brain MRI (Magnetic Resonance Imaging): Huge extra-axial disomogeneous mass with necrotic areas inside, infiltrating the meninges and compressing but not infiltrating the brain. B. Histological examination. Inner side of calvaria. A lamellar bone trabecula can be seen on the left side of the picture. A well to moderately differentiated follicular carcinoma can be appreciated with its typical histomorphology. Notice the presence of well-formed neoplastic follicular structures some of which appear to contain a dense colloid (H and E, 400×, original magnification). C. Neck CT Scan-Coronal image. A solid lesion is occupying the left lobe of the thyroid. Signs of contralateral displacement of the trachea without signs of infiltration. D. Histological examination of the thyroid tumor. H/E Magnification 40×. Multiple nests of follicular neoplasia infiltrating thickened capsule associated with neoplastic angioinvasion.

parietooccipital region. On examination the mass was firm and fixed to the skull, it measured about 8 $\mathrm{cm}$ in diameter. Systemic and neurological examination found no abnormalities. Medical history included breast surgery for cancer.

Cranial Magnetic Resonance Imaging (MRI) with intravenous gadolinium contrast medium showed a large, highly vascularized extra-axial inhomogeneous mass $(8 \times 6 \mathrm{~cm}$ in size $)$ with necrotic areas inside, infiltrating the meninges and compressing, but not infiltrating the brain (Fig. 1A).

The patient underwent surgery. Intraoperatively, the tumor appeared as a soft, red, highly vascularised mass with dural invasion. The mass was successfully and completely dissected. Postoperatively, the patient was observed in the surgical intensive care unit. She remained free from neurologic deficits. Histological examination revealed a metastatic follicular thyroid carcinoma (Fig. 1B).

The patient was then referred to our department for the treatment of the primary tumor. A neck and chest Computed Tomography (CT) scan was made that showed a $6 \times 5 \mathrm{~cm}$ mass in the left thyroid lobe with extension to the upper mediastinum (Fig. 1C). The serum thyroglobulin level was extremely high $(45.000$ $\mathrm{ng} / \mathrm{dL}$ ); the anti-thyroglobulin antibodies were negative. On June 2011, the patient underwent total thyroidectomy. The thyroid specimen weighted gr 75; on cut section, at the left lobe a solid nodule, $\mathrm{cm} 4.8$ in diameter, was present. Microscopic examination of the thyroid tumor showed a follicular neoplasm with various growth patterns: micro-macrofollicular areas 

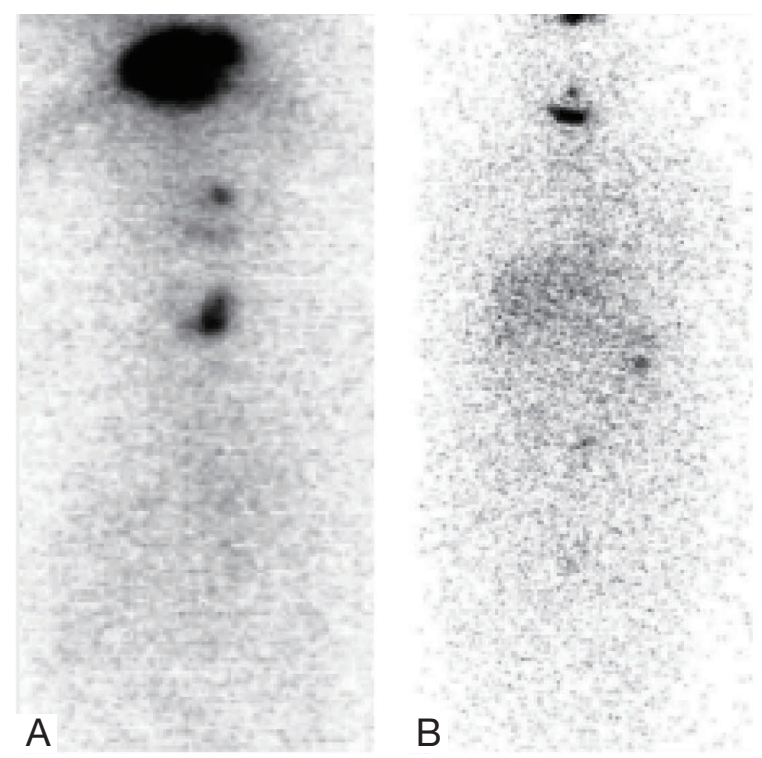

Fig. 2 Case 1

A. ${ }^{131}$ I Whole-Body Scan after the first radioiodine therapy $(7.4 \mathrm{GBq})$ : intense and large skull uptake and mild uptake in the thyroid bed. B. ${ }^{13 \mathrm{I}} \mathrm{I}$ Whole-Body Scan after second radioiodine therapy $(7.4 \mathrm{GBq})$ : marked decrease in uptake and size of the skull lesion and absence of uptake in the thyroid bed

alternating with trabecular and solid/insular areas. A thick, fibrous capsule was still evident around the nodule although completely invaded by tumor; extensive vascular invasion, within and beyond the tumor capsule, was also observed (Fig. 1D).

The histological diagnosis of follicular thyroid carcinoma with extensive capsular and vascular invasion (more than 4 foci of angioinvasion), was formulated.

The patient was then treated with $7.4 \mathrm{GBq}$ of ${ }^{131} \mathrm{I}$ after recombinant human TSH (rhTSH) stimulation. A post therapy ${ }^{131}$ I Whole Body Scan (WBS) performed a week later showed a residual uptake in the thyroid bed and intense and large uptake in the skull (Fig. 2A).

Additional $7.4 \mathrm{GBq}$ of ${ }^{131} \mathrm{I}$ were given six months later. As compared to baseline, ${ }^{131}$ I WBS scan uptake decreased dramatically (Fig. 2B), and thyroglobulin declined from $45000 \mathrm{ng} / \mathrm{mL}$ to $13.2 \mathrm{ng} / \mathrm{mL}$.

Three years after treatment she is well, on suppressive levothyroxine therapy with thyroglobulin levels of $4.5 \mathrm{ng} / \mathrm{mL}$. Brain MRI and 18FDG-PET showed no evidence of disease.

\section{Case 2}

On November 2011 a 74-year-old female with multiple slow-growing masses in the skull was admitted to the department of neurosurgery of another Hospital.

The neurological examination showed no abnormalities.

Cranial MRI with intravenous gadolinium contrast medium showed two expansile lytic lesions in the left frontal- parietal area $(6.9 \times 4.5 \times 6.6 \mathrm{~cm}$ in size $)$ and in the right parietooccipital region $(1.7 \times 2.5 \times 2.0 \mathrm{~cm}$ in size) respectively (Fig. 3A). She underwent surgical resection of the left frontal lesion only. Histological examination revealed a metastatic follicular thyroid carcinoma (Fig. 3B).

On December 2011 the patient was referred to our Unit for the treatment of the primary tumor. Clinical examination revealed an enlarged left thyroid lobe with a firm thyroid mass (about $5 \mathrm{~cm}$ diameter) that had been present for several years. A neck CT scan showed a 7 x $5 \mathrm{~cm}$ mass in the left thyroid lobe, without evidence of neck lymph node involvement (Fig. 3C). The serum thyroglobulin level was $18.000 \mathrm{ng} / \mathrm{dL}$ in the absence of anti-thyroglobulin antibodies. The patient underwent total thyroidectomy.

Upon macroscopic examination, the thyroid gland was enlarged and multinodular and weighted 182 grams. The left lobe, which was larger than the right one, was occupied by a solid macronodule measuring $7.5 \times 4.5 \times 5 \mathrm{~cm}$. Microscopic examination showed a follicular neoplasm with microfollicular growth pattern alternating with trabecular and solid areas; extensive vascular invasion, within and beyond the tumor capsule, was observed. The diagnosis was poorly differentiated follicular thyroid carcinoma (Fig. 3D)

Eight weeks after thyroidectomy she underwent radioiodine therapy $\left(7.4 \mathrm{GBq}\right.$ of $\left.{ }^{131} \mathrm{I}\right)$ after thyroid hormone withdrawal. ${ }^{131}$ I WBS performed one week after treatment detected multiple bone metastatic lesions, including an intense skull metastasis uptake (Fig. 4A). Six months later she underwent a second course of radioiodine therapy $(7.4 \mathrm{GBq})$. The ${ }^{131} \mathrm{I}$ WBS performed after completion of treatment showed a marked reduction of the bone metastases uptake, including the skull lesions (Fig. 4B) and the thyroglobulin levels declined to $422 \mathrm{ng} / \mathrm{mL}$. She received suppressive thyroxine treatment and monthly infusions of $4 \mathrm{mg}$ of zoledronic acid for her bone metastases. Two years after treatment she is well with stable metastatic bone disease. The thyroglobulin level fell further to $82 \mathrm{ng} / \mathrm{mL}$, on levothyroxine treatment. Brain MRI showed no evidence of recurrence and PET-FDG scan showed stable bone disease. 

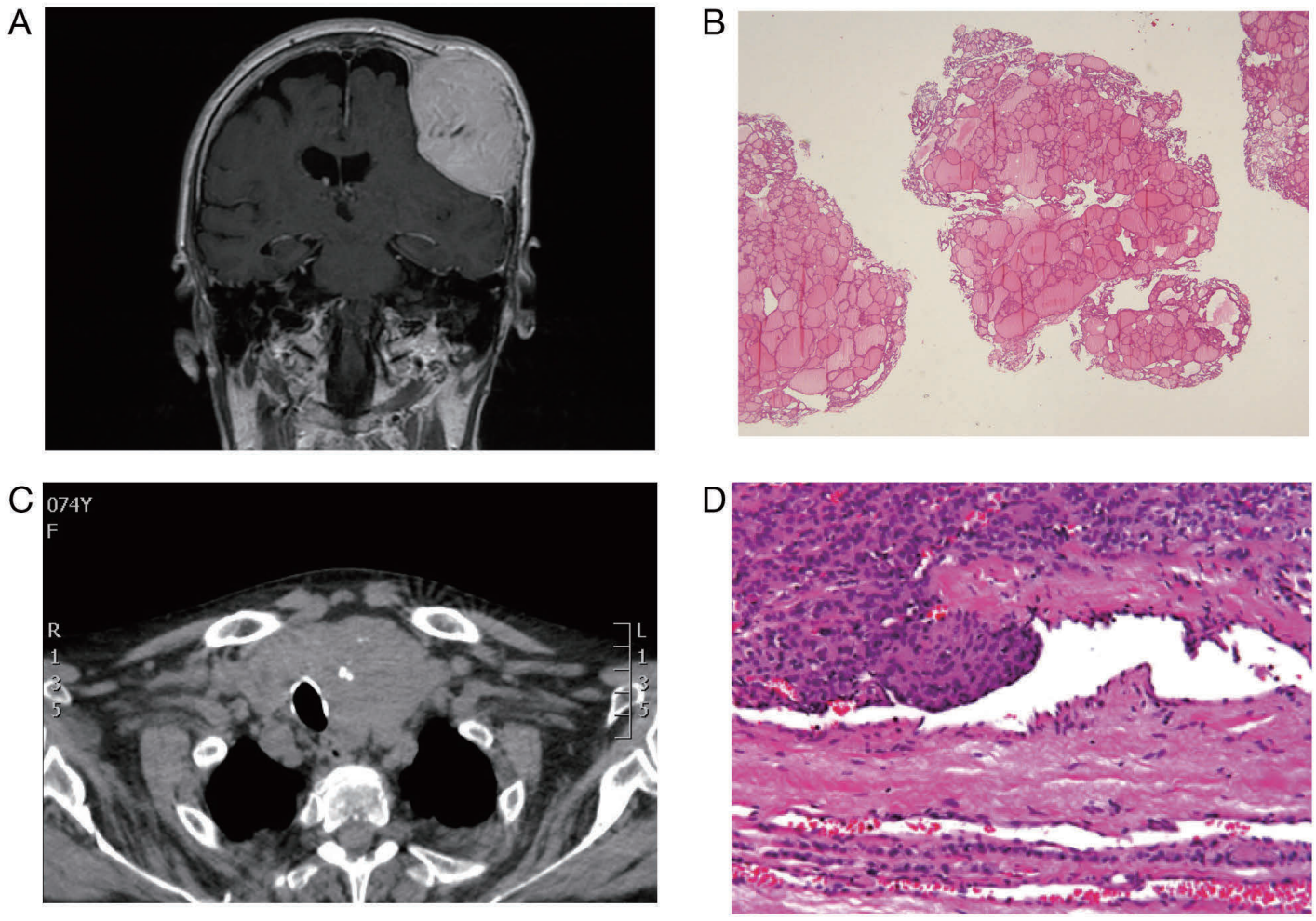

Fig. 3 Case 2

A. T1 FLAIR Brain MRI - Coronal Plane: Intracranial extradural mass compressing but not infiltrating the neural tissue. B. Histological examination. Well-differentiated follicular thyroidal neoplasia taken from the skull lesion (H/E Magnification $40 \times$ ). C. Neck CT scan. Axial image showing the large thyroid tumor of the left lobe with displacement of the trachea. D. Histological examination - Thyroid tumor. High microscopic magnification showing neoplastic infiltration of a blood vessel by the neoplasm, which firmly adheres to the vascular endothelium. ( $\mathrm{H}$ and E, 400×, original magnification).
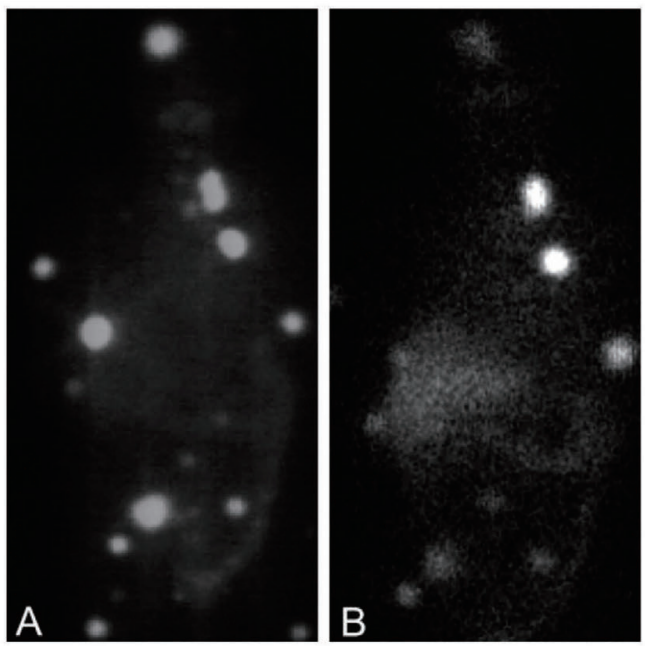

Fig. 4 Case 2

A. ${ }^{131}$ I Whole-Body Scan after the first treatment with $1.85 \mathrm{GBq}$ of ${ }^{131} \mathrm{I}$ : multiple bone metastatic lesions, including intense skull uptake. B. ${ }^{131}$ I Whole-Body Scan after the second treatment with $7.4 \mathrm{GBq}$ of 131I: Marked reduction in uptake of the bone metastases (including the skull lesion). Absence of uptake in the thyroid bed.

\section{Discussion}

Skull metastasis is a rare event in thyroid carcinoma although bone represents the second most common site of distant metastases after lung. An occurrence of 2.5\% was described in a large series of patients [6]. A small number of reports can be also found in the literature, with a prevalence of cases with follicular carcinoma over papillary carcinoma as histological subtype [7].

Indeed, skull localizations have been described mostly as recurrence, even belatedly [8] while very few cases refer to skull metastasis as presenting features of a primary thyroid cancer. When occurring in the skull, metastatic localizations of thyroid carcinoma act as asymptomatic insidious palpable mass preferentially localized in the occipital and temporal areas, and have protracted clinical course, before the diagnosis is made. In the largest reported series of skull metastasis from thyroid carcinoma, the mean period from diagnosis of thyroid tumor until discovery of the skull meta- 
stasis was 23.3 year [6].

The lesions have been reported as hyper vascular and osteolytic with involvement of subcutaneous tissue and occasional intracranial extension. At radiological study, they appear lytic and vascular. On magnetic resonance imaging (MRI), these lesions appear hyperintense on T2 weighted sequences and hypointense on unenhanced $\mathrm{T} 1$ weighted images [9].

The most common clinical sign is a palpable mass of the scalp while cranial nerve dysfunction, hemiparesis and signs of increased intracranial pressure are rare. On clinical examination, most of these metastatic lesions present as soft, painless slow-growing, even long-standing, scalp masses and are located in the occipital and temporal areas [7]. In most cases, they infiltrate the meninges and compress but do not infiltrate the brain. This kind of dural metastasis can be mistaken for meningiomas, and the definitive diagnosis is usually established only after the histopathological examination [10]. These patients, in fact, are often referred to a neurosurgeon or neurologist and after the diagnosis is established they are referred to the endocrine surgeon. In both the patients presented in this paper and in other cases reported in the literature, the levels of thyroglobulin were highly elevated at diagnosis. This serum marker can be easily and affordably performed preoperatively in the differential diagnosis of skull swellings and should be considered in the preoperative work-up.

The way of spread of thyroid carcinoma into the skull is expected via the hematogenous route. A vertebral venous plexus consisting of a valveless vascular bed is within the spinal canal and extends from the skull to the pelvis [11]. Multiple anastomoses and free connections exist between this venous plexus and the dural sinuses, the emissary veins of the skull.

Initial aggressive treatment is the crucial point in the management of metastatic thyroid carcinoma, providing the best chance to prolong survival. The combination of treatment modalities including aggressive surgical treatment, radioiodine therapy and levothyroxine suppression therapy is proven to be associated with considerable improvement of the overall survival and disease-specific survival [3]. Every effort should be made to provide surgical treatment as complete as possible even in the presence of distant metastases. Although surgical treatment of isolated distant metastases from thyroid cancer is highly recommended whenever possible [4], removal of skull metastases may be difficult due to both local spread and profuse bleeding caused by hyper vascularization [12]. Unlike the majority of other bone metastasis, skull metastasis is often suitable for surgical resection and local control of disease can be achieved in most cases. The removal of this metastatic lesions is mandatory to avoid compression of the brain and any additional destructive effect from further bone erosion. In addition, metastasis excision could enhance the effectiveness of radioiodine therapy by reducing the ${ }^{131}$ I uptake in the metastatic area.

The use of radioiodine therapy after surgery is appropriate as first-line adjuvant therapy in multiple, well differentiated distant metastases, i.e., metastases that express high levels of thyroglobulin and concentrate the radioiodine. The rationale for radioiodine treatment of metastatic disease is to destroy clinically apparent gross disease, above all, when a complete surgical resection is not attainable. Radioiodine therapy may reduce the risk of recurrence and mortality, particularly in patients with a small amount of disease that is RAI avid. Although bone metastases are often seen on ${ }^{131}$ I-scans, they generally do not uptake ${ }^{131}$ I very well; complete remission of the disease occurs in less than $10 \%$ of treated patients and partial resolution in about $35 \%$ [13].

In the cases presented herein, the skull metastases were markedly RAI avid and the patients expressed high levels of thyroglobulin; in such cases the postoperative radioiodine therapy was considered as the first and more appropriate choice of treatment. In both cases, a dramatic reduction in the thyroglobulin level was obtained along with a marked decrease of radioiodine uptake. Zoledronic acid was administered to our second patient with multiple bone metastases, for the prevention of bone complications. Therapy with zoledronic acid have been reported to be safe, welltolerable, and effective for the palliation of symptomatic bone metastases in differentiated thyroid cancer [14]. In the presented case the treatment was well tolerated, and although a antitumor action, as evidenced by decreasing the size, was not observed, the patient achieved stability of bone lesions and improvement of quality of life.

Treatment of metastatic differentiated thyroid cancer may also include external beam radiotherapy (ERBT). Although the role of EBRT has no real benefit as postoperative treatment in patients with well-differentiated thyroid cancer [15], it may play a role to improve local control in selected cases. Therefore, ERBT should be 
reserved for patients with metastatic thyroid cancer that do not concentrate the radioiodine, for bone metastases that are not amenable to surgical resection, for residual tumor after surgery and radioiodine therapy, in whom the chance to eradicate the tumor is poor unless the absorbed dose of radiation is too high. In these cases, EBRT can improve local control and palliate pain [16].

In the recent years, several advances have been made in the field of molecular biology of thyroid cancer, allowing to design appropriate targeted therapies for patients with radioiodine-refractory, locally advanced, or metastatic differentiated thyroid carcinoma [17]. Currently the only approved drug in DTC is sorafenib, even if, in the next future other tyrosine kinases inhibitors might be used in the clinical practice, having shown a good activity in clinical trials, even if employed as second and third line of therapy [18]. Although several trials have demonstrated the efficacy of tyrosine kinase inhibitors in the treatment of refractory and progressive thyroid cancer, bone metastases from thyroid carcinoma seem to be poorly responsive to TKI therapy. In thyroid cancer metastatic to bones, locoregional treatments (ie cementoplasties, percutaneous procedures using radiofrequency ablation, spinal decompression), are more effective than systemic targeted therapy and should be considered as first option, when possible [19].

The clinical approach to metastatic thyroid cancer should rely on a multidisciplinary teamwork carefully tailoring treatment for each individual patient, based on disease presentation and prognosis. In thyroid cancer with distant metastases, the definitive cure is rarely achievable. Therefore, the main goal of management is the local control of disease, management of symptoms and improvement in survival.

\section{Consent}

Written informed consent was obtained from the patient for publication of these Case reports and any accompanying images. A copy of the written consent is available for review by the Editor of this journal.

\section{Competing interests}

There is no competing interest.

\section{Acknowledgements}

We wish to acknowledge Dr Gaetano Corazzelli, who read through the manuscript and revised it critically.

\section{References}

1. Grebe SK, Hay ID (1995) Follicular thyroid cancer. Endocrinol Metab Clin North Am 24: 761-801.

2. Sampson E, Brierley JD, Le LW, Rotstein L, Tsang RW (2007) Clinical management and outcome of papillary and follicular (differentiated) thyroid cancer presenting with distant metastasis at diagnosis. Cancer 110: 14511456.

3. Haugen BR, Kane MA (2010) Approach to the thyroid cancer patient with extracervical metastases. J Clin Endocrinol Metab 95: 987-993.

4. Haq M, Harmer C (2005) Differentiated thyroid carcinoma with distant metastases at presentation: prognostic factors and outcome. Clin Endocrinol (Oxf) 63: 87-93.

5. Mihailovic J, Stefanovic L, Malesevic M (2007) Differentiated thyroid carcinoma with distant metastases: probability of survival and its predicting factors. Cancer Biother Radiopharm 22: 250-255.

6. Nagamine Y, Suzuki J, Katakura R, Yoshimoto T, Matoba N, Takaya K (1985) Skull metastasis of thyroid carcinoma. Study of 12 cases. J Neurosurg 63: 526-531.
7. Miyawaki S, Yamazaki R, Harada T, Takanashi S, Nagashima T, et al. (2007) Skull metastasis of thyroid papillary carcinoma. J Clin Neurosci 14: 481-484.

8. Bhandary SK, Bhat VS, Shenoy MS (2010) A rare case of skull base metastasis from follicular carcinoma of thyroid. Indian J Surg Oncol 1: 334-336.

9. Yildirim T, Kayaselcuk F, Erdogan B, Aydın V, Sen O, et al. (2004) Solitary skull metastasis of follicular thyroid carcinoma:CT and MR findings. Eur J Rad Extra 51: 51-55.

10. Portocarrero-Ortiz L, Garcia-Lopez R, Romero-Vargas S, Padilla JA, Gomez-Amador JL, et al. (2009) Thyroid follicular carcinoma presenting as skull and dural metastasis mimicking a meningioma: a case report. $J$ Neurooncol 95: 281-284.

11. Batson OV (1940) The Function of the Vertebral Veins and Their Role in the Spread of Metastases. Ann Surg 112: 138-149.

12. Akdemir I, Erol FS, Akpolat N, Ozveren MF, Akfirat M, et al. (2005) Skull metastasis from thyroid follicular carcinoma with difficult diagnosis of the primary lesion. 
Neurol Med Chir (Tokyo) 45: 205-208.

13. Maxon HR 3rd, Englaro EE, Thomas SR, Hertzberg VS, Hinnefeld JD, et al. (1992) Radioiodine-131 therapy for well-differentiated thyroid cancer--a quantitative radiation dosimetric approach: outcome and validation in 85 patients. $J$ Nucl Med 33: 1132-1136.

14. Orita Y, Sugitani I, Toda K, Manabe J, Fujimoto Y (2011) Zoledronic acid in the treatment of bone metastases from differentiated thyroid carcinoma. Thyroid 21: 31-35.

15. Samaan NA, Schultz PN, Hickey RC, Goepfert H, Haynie TP, et al. (1992) The results of various modalities of treatment of well differentiated thyroid carcinomas: a retrospective review of 1599 patients. J Clin Endocrinol Metab 75: 714-720.

16. O'Connell ME, A'Hern RP, Harmer CL (1994) Results of external beam radiotherapy in differentiated thy- roid carcinoma: a retrospective study from the Royal Marsden Hospital. Eur J Cancer 30A: 733-739.

17. Leboulleux S, Bastholt L, Krause T, de la Fouchardiere C, Tennvall J, et al. (2012) Vandetanib in locally advanced or metastatic differentiated thyroid cancer: a randomised, double-blind, phase 2 trial. Lancet Oncol 13: 897-905.

18. Perri F, Pezzullo L, Chiofalo M G, Lastoria S, Di Gennaro F, et al. (2014) Targeted therapy: A new hope for thyroid carcinomas. Crit Rev Oncol Hematol [Epub ahead of print].

19. Massicotte MH, Brassard M, Claude-Desroches M, Borget I, Bonichon F, et al. (2014) Tyrosine kinase inhibitor treatments in patients with metastatic thyroid carcinomas: a retrospective study of the TUTHYREF network. Eur J Endocrinol 8: 575-82. 